\title{
INTERAÇÃO SILÍCIO COM INSETICIDA REGULADOR DE CRESCIMENTO NO MANEJO DA LAGARTA-DO-CARTUCHO Spodoptera frugiperda (J. E. Smith, 1797) (LEPIDOPTERA: NOCTUIDAE) EM MILHO ${ }^{1}$
}

\author{
Interaction of silicon with growth regulating insecticide in the management of fall \\ armyworm Spodoptera frugiperda (J. E. SMITH, 1797) \\ (Lepidoptera: Noctuidae) in corn plants
}

\author{
Danila Kelly Pereira Neri², Jair Campos Moraes $^{3}$, Marcio Augusto Gavino ${ }^{4}$
}

\begin{abstract}
RESUMO
Com o objetivo de avaliar o efeito do silício aplicado via solo e foliar, bem como sua interação com o inseticida regulador de crescimento (lufenuron) no manejo de Spodoptera frugiperda em plantas de milho, foi realizado um ensaio em casa-de-vegetação e em laboratório, constando de nove tratamentos com cinco repetições. No laboratório do Departamento de Entomologia da Universidade Federal de Lavras-MG, avaliou-se a preferência das lagartas por folhas destacadas de plantas de milho provenientes dos diferentes tratamentos, bem como o consumo e a mortalidade dessa praga. Em casa-de-vegetação, foram avaliadas a intensidade das injúrias provocadas pelas lagartas nas folhas, utilizando uma escala visual de danos proposta por Davis \& Williams (1989), bem como o número e a biomassa das lagartas vivas. Pelos resultados pode-se concluir que os tratamentos não afetaram a preferência da lagartado-cartucho em teste de livre escolha. A interação silício e lufenuron no manejo de $S$. frugiperda é positiva em relação ao inseticida isolado, provavelmente devido a resistência mecânica conferida pelo silício as folhas.
\end{abstract}

Termos para indexação: Insecta, resistência induzida e lufenuron.

\begin{abstract}
The objective of this work was to evaluate the effect of sprayed silicon via soil and leaf as well as its interaction with growth regulating insecticide (lufenuron) in the management of Spodoptera frugiperda on corn plants. The trials were carried out in greenhouse and laboratory conditions and consisting of nine treatments with five replicates. In the laboratory, the preference of fall armyworms on detached leaves of corn plants from different treatments was evaluated, as well as the consumption and mortality of this pest. In the greenhouse, the damage caused by the insect on the leaves were evaluated by using a visual scale of injuries proposed by Davis \& Williams (1989). In the greenhouse on the control treatment, both the number and weight of the larval were also determined. According to the results, silicon, the insecticide lufenuron and the interaction between silicon and lufenuron did not affect the preference of fall armyworm in a free choice test. The silicon x lufenuron interaction is positive in the management of $S$. frugiperda when compared to the insecticide only, probably due to a mechanical resistance by the silicon on the leaves surface.
\end{abstract}

Index terms: Insecta, induced resistance, lufenuron.

(Recebido para publicação em 22 de setembro de 2004 e aprovado em 10 de junho de 2005)

\section{INTRODUÇÃO}

O milho (Zea mays L.) é um dos cereais mais importantes do mundo por constituir a base da alimentação humana e animal (SILVA, 1995). A produção de milho tende a se expandir fortemente para suprir a demanda gerada pelo crescimento populacional e, principalmente, pelo consumo de fontes de energia renováveis, estabelecendo, assim, a era da agricultura energética (SILVA, 2004).

$\mathrm{Na}$ condução da cultura muitos problemas são observados, podendo ser relacionados à precária situação de renda dos agricultores, à baixa disponibilidade de capital para a agricultura, ao baixo nível de tecnologia adotado e à incidência de pragas, que atacam a planta de milho praticamente em todas as fases do seu ciclo, ocasionando prejuízos de ordem quantitativa e qualitativa (BOIÇA JUNIOR et al., 1996).

A principal praga do milho no Brasil é Spodoptera frugiperda (J. E. Smith, 1797) (Lepidoptera: Noctuidae), que ocorre em todo o ciclo da cultura causando consideráveis perdas na produção. A dimensão das perdas provocadas pode variar em função da cultivar utilizada, da fase fenológica, do sistema de produção empregado e do local de plantio (SARMENTO et al., 2002).

${ }^{1}$ Parte da Dissertação de Mestrado do primeiro autor - Departamento de Entomologia - Universidade Federal de Lavras/UFLA.

${ }^{2}$ Engenheira Agrônoma M.Sc. - Departamento de Entomologia - Universidade Federal de Lavras/UFLA - Cx. P. 3037 - 37.200-000 - Lavras,MG danilaneri@yahoo.com.br

${ }^{3}$ Professor Doutor do Departamento de Entomologia - Universidade Federal de Lavras/UFLA, Cx. P. 3037 - 37200-000 - Lavras,MG - jcmoraes@ufla.br ${ }^{4}$ Aluno do $10^{\circ}$ período da graduação em Engenharia Agronômica - Departamento de Entomologia - Universidade Federal de Lavras - Cx. P. 3037 37200-000 - Lavras,MG. 
O controle da lagarta-do-cartucho tem sido realizado principalmente pelo uso de inseticidas sintéticos, sendo que o uso indiscriminado desses pode levar ao surgimento da resistência a campo. O lufenuron é o ingrediente ativo de um novo inseticida do grupo dos inibidores da biossíntese de quitina utilizado para o controle de $S$. frugiperda na cultura do milho. (SCHMIDT, 2002). A utilização de variedades resistentes associadas ao controle químico reduz o número de tratamentos e/ou a dose do inseticida empregado (VENDRAMIM, 1990).

Estudos recentes têm mostrado que o silício pode estimular o crescimento e a produção vegetal por meio de várias ações indiretas, propiciando proteção contra fatores abióticos, como estresse hídricos, toxidez de alumínio, ferro, entre outros, e bióticos, como a incidência de insetos-praga (EPSTEIN, 1994). Dessa forma, a aplicação de silício na cultura do milho poderá elevar o grau de resistência das plantas e, conseqüentemente, reduzir a infestação e os prejuízos causados por $S$. frugiperda.

Objetivou-se com este trabalho avaliar o efeito do silício aplicado via solo e foliar, bem como sua interação com o inseticida regulador de crescimento (lufenuron) no manejo de $S$. frugiperda em plantas de milho.

\section{MATERIAL E MÉTODOS}

\section{Metodologia geral}

O experimento foi conduzido em casa-de-vegetação e no laboratório do Departamento de Entomologia/UFLA, no período de março a abril de 2004.

Semearam-se 4 sementes de milho híbrido AS-3466 Precoce (Agroeste ${ }^{\hat{a}}$ ) por vaso com capacidade para 5 litros de substrato, composto por terra de barranco adubada com esterco de curral (2:1) e $15 \mathrm{~g}$ de NPK (4-14-8), além do agrosilício, que foi utilizado para alguns tratamentos. A umidade do substrato foi mantida por meio de irrigações diárias. Após 10 dias da emergência, procedeu-se o desbaste das plantas excedentes deixando-se apenas a planta mais vigorosa por vaso. Cada parcela experimental foi constituída por dois vasos, sendo um utilizado no experimento em laboratório e o outro, na casa-de-vegetação.

Previamente à implantação do experimento, uma amostra do solo foi submetida à análise química pelo Laboratório de Análise de Solo do Departamento de Ciência dos solos/UFLA (Tabela 1).

Os vasos foram dispostos ao acaso sobre bancadas metálicas em casa-de-vegetação, envolvendo nove tratamentos e cinco repetições. Os tratamentos testados foram: $\mathrm{T} 1$ = aplicação de $2 \mathrm{~g}$ de agrosilício/kg de solo incorporada ao substrato antes do plantio; T2 = aplicação foliar de ácido silícico $\left(0,5 \%\right.$ de $\left.\mathrm{SiO}_{2}\right)$ aos 10 dias após a emergência; $\mathrm{T} 3$ = aplicação de $2 \mathrm{~g}$ de agrosilício/kg de solo incorporada ao substrato antes do plantio + uma pulverização com o inseticida lufenuron (Match ${ }^{\hat{a}} \mathrm{CE}$ ) na dose 7,5 g i.a./ha, um dia antes da liberação das lagartas; $\mathrm{T} 4$ = aplicação de $2 \mathrm{~g}$ de agrosilício/kg de solo incorporada ao substrato antes do plantio + uma pulverização com o inseticida lufenuron na dose de $15 \mathrm{~g}$ i.a./ha (dose recomendada) um dia antes da liberação das lagartas; T5 = aplicação foliar de ácido silícico $\left(0,5 \%\right.$ de $\left.\mathrm{SiO}_{2}\right)$ aos 10 dias após a emergência + uma pulverização com o inseticida lufenuron na dose $7,5 \mathrm{~g}$ i.a./ha, um dia antes da liberação das lagartas; $\mathrm{T} 6=$ aplicação foliar de ácido silícico $(0,5 \%$ de $\mathrm{SiO}_{2}$ ) aos 10 dias após a emergência + uma pulverização com o inseticida lufenuron na dose $15 \mathrm{~g}$ i.a./ha, um dia antes da liberação das lagartas; T7 = pulverização com o inseticida lufenuron na dose $7,5 \mathrm{~g}$ i.a./ha, um dia antes da liberação das lagartas; T8 = pulverização com o inseticida lufenuron na dose $15 \mathrm{~g}$ i.a./ha, um dia antes da liberação das lagartas; $\mathrm{T} 9=$ testemunha (sem silício e sem inseticida).

Quando as plantas atingiram o estádio 2 de desenvolvimento fisiológico ( 3 a 4 folhas), período em que a cultura se encontra mais susceptível ao ataque desta praga (CRUZ \& TURPIN, 1982), iniciaram-se os testes para avaliação de resistência com lagartas, provenientes da criação de manutenção, que tinham um dia de idade, e que estavam sob dieta artificial desde a eclosão (BOGORNI \& VENDRAMIM, 2003)

TABELA 1 - Análise química do solo utilizado no experimento, realizada pelo Laboratório de Análises de Solos do Departamento de Ciência do Solo da UFLA. 2004.

\begin{tabular}{|c|c|c|c|c|c|c|c|c|c|c|c|}
\hline $\mathbf{P h}$ & $\mathbf{P}$ & $\mathbf{K}$ & $\mathrm{Ca}^{2+}$ & $\mathbf{M g}^{2+}$ & $\mathbf{A l}^{3+}$ & $\mathrm{H}+\mathrm{Al}$ & SB & (T) & V & $\mathbf{m}$ & P-rem \\
\hline $\mathrm{H}_{2} \mathrm{O}$ & \multicolumn{2}{|c|}{$\mathrm{mg} / \mathrm{dm}^{3}$} & & \multicolumn{5}{|c|}{ - } & \multicolumn{2}{|c|}{---- \% ---- } & $\mathrm{mg} / \mathrm{L}$ \\
\hline 5,7 & 2,0 & 14 & 2,4 & 0,9 & 0,0 & 4,0 & 3,3 & 7,3 & 45,5 & 0,0 & 11,5 \\
\hline
\end{tabular}

Ciênc. agrotec., Lavras, v. 29, n. 6, p. 1167-1174, nov./dez., 2005 
Teste de livre escolha em folhas destacadas de plantas de milho

A preferência alimentar de $S$. frugiperda foi avaliada em testes de livre escolha em laboratório. As seções foliares, retângulos de $1,5 \mathrm{~cm}$ x $2,0 \mathrm{~cm}$, foram obtidas da quarta folha totalmente expandida das plantas de cada tratamento, as quais foram dispostas circularmente em placas de Petri (15 cm de diâmetro), que continham papel filtro umedecido com água destilada, formando-se, assim, uma arena - modificado de Silveira et al. (1998). Imediatamente após, 27 lagartas foram liberadas no centro das placas, vedando-se em seguida com filme de PVC. As placas foram colocadas em câmara climatizada regulada a $25 \pm 2{ }^{\circ} \mathrm{C}$, UR de $70 \pm$ $10 \%$ e fotofase de 12 horas. O número de lagartas sobre as folhas de cada tratamento foi registrado após 24 e 48 $\mathrm{h}$ da liberação.

Utilizou-se o delineamento em blocos ao acaso, com dez repetições. Os dados obtidos foram transformados em $\sqrt{(x+0,01)}$ e submetidos à análise de variância, sendo as médias comparadas pelo teste de Duncan a 5\% de significância.

Teste de preferência sem chance de escolha em folhas destacadas de plantas de milho

Na execução desse bioensaio, uma seção foliar de 6 $\mathrm{cm}$ de comprimento da quarta folha totalmente expandida das plantas de cada tratamento foi colocada numa placa de Petri (15 cm de diâmetro) contendo, no fundo, um papel filtro umedecido com água destilada para manter a turgescência da folha de milho. Cada placa recebeu 20 lagartas e a mortalidade foi avaliada 24,48 e 72 h após. Adotou-se o delineamento inteiramente casualizado com cinco repetições.

O consumo foliar foi determinado após $48 \mathrm{~h}$ do fornecimento das folhas de milho de cada tratamento, quando o segmento foliar foi recolhido e substituído por um outro pertencente, agora, a quinta folha. Quantificou-se, dessa forma, o consumo das lagartas após 48 e 96 h da instalação do experimento. De cada tratamento deixou-se uma alíquota para poder medir a perda da umidade. O consumo alimentar foi obtido pela diferença do peso foliar entre a porção oferecida e a sobra.

Os dados foram submetidos à análise de variância, sendo os de mortalidade transformados em $\operatorname{arco-seno}(\sqrt{x / 100})$ antes de proceder a análise. As médias foram comparadas pelo teste de Duncan a 5\% de significância.

\section{Avaliação do dano de $S$. frugiperda em plantas de milho}

Para avaliar os danos, os tratamentos foram infestados artificialmente com 10 lagartas, sendo colocadas na quarta folha com auxílio de um pincel. Após 10 dias da infestação, foi realizada uma avaliação do ataque, utilizando uma escala visual de danos proposta por Davis \& Williams (1989) (Tabela 2), sendo a nota média de danos obtida das notas atribuídas por quatro avaliadores.

Após o surgimento de fezes no interior do cartucho das plantas do tratamento testemunha (característica da presença da praga no cartucho), as lagartas de todos os tratamentos foram contadas e levadas ao laboratório para a determinação da biomassa.

Foi utilizado o delineamento inteiramente casualizado com cinco repetições. Os dados obtidos foram submetidos a análise de variância, sendo os referentes ao número de lagartas presentes nos diferentes tratamentos transformados em. As médias foram comparadas pelo teste de Duncan a 5\% de significância.

\section{RESULTADOS E DISCUSSÃO}

\section{Teste de livre escolha com folhas destacadas de plantas de milho}

Não houve diferença entre os tratamentos, na preferência das lagartas de primeiro instar em teste de livre escolha, após 24 e 48 horas da liberação (Tabela 3). Nas avaliações feitas após 24 e 48 horas da liberação das lagartas, constatou-se uma faixa de $3,5 \pm 0,7$ a $1,7 \pm 0,5$ e $3,0 \pm 0,6$ a $1,1 \pm 0,3$ lagartas/ tratamento, respectivamente. Provavelmente, o período de avaliação não foi suficiente para detectar diferenças entre os tratamentos. Contudo, um maior período de avaliação poderia acarretar uma mortalidade excessiva de lagartas devido aos tratamentos com inseticida e a ocorrência de canibalismo que é comum nessa espécie, como constatado por Goussain et al. (2002) quando aplicaram uma solução de silicato de sódio via solo em plantas de milho. 
TABELA 2 - Escala visual de danos para Spodoptera frugiperda (DAVIS \& WILLIAMS, 1989).

\begin{tabular}{cl}
\hline Nota & \multicolumn{1}{c}{ Descrição } \\
\hline 0 & Nenhum dano nas folhas \\
1 & Perfurações diminutas em algumas folhas \\
2 & Pequena quantidade de perfurações arredondadas em algumas folhas \\
3 & Perfurações arredondadas em várias folhas \\
4 & Perfurações arredondadas e lesões em algumas folhas \\
5 & Lesões em várias folhas \\
6 & Grandes lesões em várias folhas \\
7 & Grandes lesões e porções comidas (dilaceradas) em algumas folhas \\
8 & Grandes lesões e porções comidas (dilaceradas) em várias folhas \\
9 & Grandes lesões e porções comidas (dilaceradas) na maioria das folhas \\
\hline
\end{tabular}

TABELA 3 - Número de lagartas de Spodoptera frugiperda (média \pm EP) em seção foliar de milho nos diferentes tratamentos em teste de livre escolha. UFLA. 2004.

\begin{tabular}{lcc}
\hline \multirow{2}{*}{ Tratamentos } & \multicolumn{2}{c}{ Número de lagartas } \\
\cline { 2 - 3 } & $\mathbf{2 4} \mathbf{~ h}$ & $\mathbf{4 8} \mathbf{~ h}$ \\
\hline Agrosilício & $2,2 \pm 0,4 \mathrm{a}$ & $1,2 \pm 0,3 \mathrm{a}$ \\
Ácido silícico & $2,8 \pm 0,5 \mathrm{a}$ & $3,0 \pm 0,6 \mathrm{a}$ \\
Agrosilício e lufenuron (7,5 g i.a./ha) & $2,3 \pm 0,4 \mathrm{a}$ & $2,1 \pm 0,5 \mathrm{a}$ \\
Agrosilício e lufenuron (15 g i.a./ha) & $3,5 \pm 0,7 \mathrm{a}$ & $2,6 \pm 0,4 \mathrm{a}$ \\
Ácido silícico e lufenuron (7,5 g i.a./ha) & $2,6 \pm 0,6 \mathrm{a}$ & $2,1 \pm 0,5 \mathrm{a}$ \\
Ácido silícico e lufenuron (15g i.a./ha) & $2,4 \pm 0,9 \mathrm{a}$ & $2,3 \pm 0,6 \mathrm{a}$ \\
Lufenuron (7,5 g i.a./ha) & $1,7 \pm 0,5 \mathrm{a}$ & $1,1 \pm 0,3 \mathrm{a}$ \\
Lufenuron (15 g i.a.ha) & $2,7 \pm 0,9 \mathrm{a}$ & $1,8 \pm 0,4 \mathrm{a}$ \\
Testemunha & $2,5 \pm 0,5 \mathrm{a}$ & $1,7 \pm 0,4 \mathrm{a}$ \\
\hline CV (\%) & 50,1 & 50,6 \\
\hline
\end{tabular}

Médias com diferenças não-significativas pelo Teste $\mathrm{F}(\mathrm{P}>0,05)$.

Teste de preferência sem chance de escolha em folhas destacadas de plantas de milho

Observa-se diferença significativa entre os tratamentos, com referência à mortalidade de lagartas após 24, 48 e 72 h da liberação (Tabela 4). Ácido silícico e lufenuron (15 g i.a./ha) proporcionaram maior mortalidade após 24 horas, diferindo significativamente dos demais tratamentos. Após 48 horas a maior mortalidade foi obtida pelo tratamento que recebeu agrosilício com lufenuron na dose de 7,5 g i.a./ha. Provavelmente, o silício conferiu uma resistência mecânica às folhas, o que dificultou a alimentação desses insetos, deixando-os mais susceptíveis à ação do inseticida, como encontrado por Goussain et al.
(2002), que verificaram efeito significativo do silício na mortalidade da lagarta-do-cartucho ao final do $2^{\mathrm{o}}$ ínstar, quando alimentadas com folhas provenientes de plantas que receberam esse mineral, apresentando o dobro da mortalidade da testemunha.

Entretanto, pelos resultados pode-se perceber que os tratamentos combinando silício e inseticida foram mais eficientes que os tratamentos isolados. Isto se justifica pelo fato do uso da combinação fornecer uma resposta mais rápida e mais eficiente no controle da lagarta-do-cartucho do milho, pois segundo Alvarez \& Datnoff (2001), o silício tem a capacidade de reduzir consideravelmente várias pragas, levando a uma redução da aplicação de inseticida. 
Na avaliação realizada após 72 horas, constatouse que todos os tratamentos com silício combinado com lufenuron apresentaram de 90 a $100 \%$ de mortalidade e os tratamentos que receberam apenas o inseticida obtiveram uma mortalidade de aproximadamente 85 a $95 \%$. Resultados semelhantes foram encontrados por Bellettini et al. (1992) ao avaliarem a utilização de produtos fisiológicos no controle da lagarta-do-cartucho. Estes autores concluíram que os inseticidas chlorfluazuron (Atabron 50CE) e diflubenzuron (Dimilin 25PM) apresentaram alta eficiência de controle de 75,2 a 97,6\% aos 03, 09 e 12 dias após a aplicação. Em outro trabalho, Gomez \& Ávila (2001) verificaram que os inseticidas teflubenzuron, triflumuron e diflubenzuron, nas doses de 10,15 e $30 \mathrm{~g} / \mathrm{ha}$, respectivamente, controlaram eficientemente as lagartas de S. frugiperda já no quarto dia após a aplicação.

Observou-se, nos tratamentos com o inseticida regulador de crescimento, um sintoma de mortalidade semelhante ao encontrado por Edomwande et al. (2000) em lagartas de primeiro ínstar de Phthorimaea operculella (Zeller, 1873) (Lepidoptera: Gelechiidae), em tubérculos de batata tratados com lufenuron; que durante a muda para o segundo ínstar as lagartas mortas exibiram um sintoma típico de benzoyilphenyl uréia, isto é, corpo murcho, falta de abrigo para a velha cutícula, redução do tamanho do corpo e ruptura do exoesqueleto.

Quanto ao consumo alimentar, verificou-se que o peso da matéria fresca consumida pelas lagartas, no período de 96 horas, foi afetado significativamente pelas concentrações do inseticida e pela forma de aplicação do silício (Tabela 5). Os resultados diferem daqueles encontrados por Goussain et al. (2002) em estudos com essa mesma praga, em teste com chance de escolha por lagartas de $3^{\circ}$ e $5^{\circ}$ instares em 24 horas, os quais não verificaram efeito significativo no consumo de folhas de plantas de milho com ou sem silício.

Nos tratamentos que receberam apenas $\mathrm{Si}$, as lagartas apresentaram maior consumo foliar, diferindo da testemunha. Esses resultados se assemelham àqueles de Oliveira et al. (1990), que ao estudarem a nutrição quantitativa da lagarta-do-cartucho em milho cultivado para três níveis de alumínio, verificaram que as folhas de milho produzidas no solo com baixo teor de $\mathrm{Al}$ mostraram-se mais adequadas como alimento, uma vez que foram menos consumidas e melhor digeridas, apresentando ainda menor custo metabólico. Conforme esses autores, houve uma tentativa de resposta compensatória, na qual a lagarta mudou seu comportamento em relação à tomada de alimento, procurando compensar as dificuldades na digestão e aproveitamento de alimento.

TABELA 4 - Porcentagem de mortalidade de lagartas de Spodoptera frugiperda (média \pm EP) nos diferentes tratamentos em teste sem chance de escolha. UFLA. 2004.

\begin{tabular}{lrrr}
\hline \multirow{2}{*}{ Tratamentos } & \multicolumn{3}{c}{ Porcentagem de mortalidade } \\
\cline { 2 - 4 } & $0,0 \pm 0,0 \mathrm{~b}$ & $\mathbf{4 8 ~ h}$ & $\mathbf{7 2 ~ h}$ \\
\hline Agrosilício & $4,0 \pm 1,1 \mathrm{~b}$ & $8,0 \pm 1,9 \mathrm{~d}$ & $9,0 \pm 1,9 \mathrm{c}$ \\
Ácido silí́cico & $0,0 \pm 0,0 \mathrm{~b}$ & $7,0 \pm 2,2 \mathrm{~d}$ & $9,0 \pm 2,1 \mathrm{c}$ \\
Agrosilício e lufenuron (7,5 g i.a./ha) & $1,0 \pm 0,4 \mathrm{~b}$ & $72,0 \pm 2,4 \mathrm{~b}$ & $100,0 \pm 0,0 \mathrm{a}$ \\
Agrosilício e lufenuron (15 g i.a./ha) & $0,0 \pm 0,0 \mathrm{~b}$ & $84,0 \pm 3,4 \mathrm{ab}$ & $93,0 \pm 0,0 \mathrm{a}$ \\
Ác. silícico e lufenuron (7,5 g i.a./ha) & $28,0 \pm 5,3 \mathrm{a}$ & $84,0 \pm 2,8 \mathrm{ab}$ & $99,0 \pm 0,4 \mathrm{ab}$ \\
Ác. silícico e lufenuron (15 g i.a./ha) & $2,0 \pm 0,9 \mathrm{~b}$ & $48,0 \pm 1,5 \mathrm{c}$ & $88,0 \pm 2,3 \mathrm{~b}$ \\
Lufenuron (7,5 g i.a./ha) & $7,0 \pm 1,3 \mathrm{~b}$ & $48,0 \pm 0,9 \mathrm{c}$ & $94,0 \pm 1,8 \mathrm{ab}$ \\
Lufenuron (15 g i.a./ha) & $1,0 \pm 0,4 \mathrm{~b}$ & $6,0 \pm 1,1 \mathrm{~d}$ & $23,0 \pm 5,4 \mathrm{c}$ \\
Testemunha & 128,0 & 24,2 & 18,8 \\
\hline CV (\%) & &
\end{tabular}

Médias seguidas da mesma letra, na coluna, não diferem estatisticamente entre si pelo Teste de Duncan $(\mathrm{P} \leq 0,05)$. 
TABELA 5 - Consumo alimentar (g) de lagartas de Spodoptera frugiperda (média \pm EP) nos diferentes tratamentos, em teste sem chance de escolha, após 96 h. UFLA. 2004.

\begin{tabular}{lc}
\hline Tratamentos & Consumo (g) \\
\hline Agrosilício & $0,5 \pm 0,03 \mathrm{a}$ \\
Ácido silí́cico & $0,5 \pm 0,02 \mathrm{a}$ \\
Agrosilício e lufenuron (7,5 g i.a./ha) & $0,2 \pm 0,02 \mathrm{c}$ \\
Agrosilício e lufenuron (15 g i.a./ha) & $0,1 \pm 0,26 \mathrm{~cd}$ \\
Ác. silícico e lufenuron (7,5 g i.a./ha) & $0,1 \pm 0,01 \mathrm{~d}$ \\
Ác. silícico e lufenuron (15 g i.a./ha) & $0,2 \pm 0,25 \mathrm{c}$ \\
Lufenuron (7,5 g i.a./ha) & $0,1 \pm 0,03 \mathrm{~cd}$ \\
Lufenuron (15 g i.a./ha) & $0,1 \pm 0,01 \mathrm{~d}$ \\
Testemunha & $0,3 \pm 0,06 \mathrm{~b}$ \\
\hline CV $(\%)$ & 31,8 \\
\hline
\end{tabular}

Médias seguidas da mesma letra, na coluna, não diferem estatisticamente entre si pelo Teste de Duncan ( $\mathrm{P} \leq 0,05)$.

\section{Avaliação do dano de $S$. frugiperda em plantas de milho}

As notas atribuídas aos danos nas folhas devido à alimentação da $S$. frugiperda e, o número de lagartas presentes nas plantas apresentaram efeito significativo, no entanto, o mesmo não foi constatado na biomassa das lagartas (Tabela 6).

No que se refere aos danos provocadas pelas lagartas presentes nas plantas de milho nos diferentes tratamentos, os maiores danos encontrados foram nas plantas que receberam apenas silício (agrosilício e ácido silícico) e na testemunha, que foram, em média, de 7,9; 7,0 e 6,7 , respectivamente. Isto indica que ao se utilizar uma fonte de silício, as lagartas provavelmente fogem para a região do cartucho do milho onde se localizam as folhas mais tenras, uma vez que a sílica confere textura à epiderme, constituindo barreira mecânica que dificulta a alimentação das lagartas, conforme constatado por Busato et al. (2002) e Goussain et al. (2002), ao observarem desgaste nos dentes da mandíbula das lagartas por ocasião da alimentação em folhas de milho contendo silício.

Já os tratamentos que receberam dose de $15 \mathrm{~g}$ i.a./ ha do inseticida, seja isolado ou em conjunto com silício, apresentaram menores danos. O efeito intermediário foi constatado quando se utilizou silício associado com a dose de 7,5 g i.a./ha do lufenuron.

O número de lagartas encontradas nas plantas de milho nos diferentes tratamentos foi significativo e semelhante às notas de danos, o que aumenta a confiabilidade do método proposto por Davis \& Williams (1989), utilizado na avaliação do experimento.

O reduzido número de lagartas presentes nas plantas que receberam inseticidas se deve a alta eficiência do inseticida lufenuron, o que confirma o relato de Gomez \& Ávila (2001) de que os inseticidas reguladores de crescimento podem controlar eficientemente as lagartas de $S$. frugiperda já no quarto dia após a aplicação.

De maneira geral a interação silício e o inseticida lufenuron foi positiva; contudo, outros testes são necessários para uma resposta conclusiva sobre o emprego desta técnica. Experimentos a campo, com infestação natural, e maior redução nas dosagens do inseticida seriam fundamentais; mesmo assim, os resultados são satisfatórios, considerando que as infestações com $S$. frugiperda foram artificiais, com número inicial de lagartas muito maior que o geralmente encontrado no campo. 
TABELA 6 - Notas dos danos, número e biomassa de lagartas de Spodoptera frugiperda (média \pm EP) nos diferentes tratamentos. UFLA. 2004.

\begin{tabular}{lccc}
\hline Tratamentos & $\begin{array}{c}\text { Nota de } \\
\text { dano }\end{array}$ & $\begin{array}{c}\text { Número de } \\
\text { lagartas }\end{array}$ & $\begin{array}{c}\text { Biomassa de } \\
\text { lagartas (g) }\end{array}$ \\
\hline Agrosilício & $7,9 \pm 0,5 \mathrm{a}$ & $2,8 \pm 0,2 \mathrm{a}$ & $0,035 \pm 0,007 \mathrm{a}$ \\
Ácido silí́cico & $7,0 \pm 0,4 \mathrm{a}$ & $2,4 \pm 0,2 \mathrm{a}$ & $0,033 \pm 0,004 \mathrm{a}$ \\
Agrosilício e lufenuron (7,5 g i.a./ha) & $4,4 \pm 0,8 \mathrm{~b}$ & $0,8 \pm 0,2 \mathrm{~b}$ & $0,046 \pm 0,007 \mathrm{a}$ \\
Agrosilício e lufenuron (15 g i.a./ha) & $1,1 \pm 0,7 \mathrm{c}$ & $0,0 \pm 0,0 \mathrm{c}$ & - \\
Ácido silícico e lufenuron (7,5 g i.a./ha) & $3,5 \pm 0,8 \mathrm{~b}$ & $0,6 \pm 0,1 \mathrm{~b}$ & $0,051 \pm 0,006 \mathrm{a}$ \\
Ácido silícico e lufenuron (15 g i.a./ha) & $1,1 \pm 0,1 \mathrm{c}$ & $0,0 \pm 0,0 \mathrm{c}$ & - \\
Lufenuron (7,5 g i.a./ha) & $1,4 \pm 0,1 \mathrm{c}$ & $0,0 \pm 0,0 \mathrm{c}$ & - \\
Lufenuron (15 g i.a./ha) & $1,5 \pm 0,1 \mathrm{c}$ & $0,0 \pm 0,0 \mathrm{c}$ & - \\
Testemunha & $6,7 \pm 0,1 \mathrm{a}$ & $3,0 \pm 0,1 \mathrm{a}$ & $0,045 \pm 0,006 \mathrm{a}$ \\
\hline CV (\%) & 24,2 & 42,4 & 34,1 \\
\hline
\end{tabular}

Médias seguidas da mesma letra, na coluna, não diferem estatisticamente entre si pelo Teste de Duncan $(\mathrm{P} \leq 0,05)$.

\section{CONCLUSÕES}

As aplicações de silício e de lufenuron, isolados ou em conjunto, não afetaram a preferência de $S$. frugiperda em teste de livre escolha.

A interação silício e lufenuron no manejo de $S$. frugiperda foi positiva, com possibilidades de redução da dose do inseticida químico pela metade.

\section{REFERÊNCIAS BIBLIOGRÁFICAS}

ALVAREZ, J.; DATNOFF, L. E. The economic potential of silicon for integrated management and sustainable rice production. Crop Protection, Guildford, n. 20, p. 43-48, 2001.

BELLETTINI, S.; BELLETTINI, N. M. T.; HIRAI, L. T.; MOREIRA, E. M.; ZANARDO, M. C.; KOBA, W. M. Utilização de produtos fisiológicos no controle da "lagartado-cartucho-do-milho", Spodoptera frugiperda (J. E. Smith, 1797) (Lep., Noctuidae). Anais da Sociedade Entomológica do Brasil, Jaboticabal, v. 21, n. 3, p. 262-266, 1992.

BOGORNI, P. C.; VENDRAMIM, J. D. Bioatividade de estratos aquosos de Trichilia spp. sobre Spodoptera frugiperda (J. E. Smith) (Lepidoptera: Noctuidade) em milho. Neotropical Entomology, Londrina, v. 32, n. 4, p. 665-669, out./dez. 2003.

BOIÇA JUNIOR, A. L.; LARA, F. M.; LUCCIN, L. M.; COSTA, G. M. Avaliação dos efeitos da adubação em milho sobre a incidência de Spodoptera frugiperda (J. E. Smith, 1797), Helicoverpa zea (Boddie, 1850) e Sitophilus zeamais (Mots., 1855). Cultura Agronômica, Ilha Solteira, v. 5, n. 1, p. 39-50, 1996.

BUSATO, G. R.; GRÜTZMACHER, A. D.; GARCIA, M. S.; GILO, F. P.; MARTINS, A. F. Consumo e utilização de alimento por Spodoptera frugiperda (J. E. Smith) (Lepidoptera: Noctuidade) originária de diferentes regiões do Rio Grande do Sul, das culturas do milho e do arroz irrigado. Neotropical Entomology, Londrina, v. 31, n. 4, 2002.

CRUZ, I.; TURPIN, F. T. Efeito da Spodoptera frugiperda em diferentes estágios de crescimento da cultura do milho. Pesquisa Agropecuária Brasileira, Brasília, v. 17, n. 3, p. 355-359, mar. 1982.

DAVIS, F. M.; WILLIAMS, W. P. Methods used to screen maize for and to determine machanisms of resistance to the southwestern corn borer and fall armyworm. In: INTERNATIONAL SYMPOSIUM ON METHODOLOGIES FOR DEVELOPING HOST PLANT RESISTANCE TO MAIZE INSECT, 1989, México. Proceedings... México: [s.n.], 1989. p. 101-108.

EDOMWANDE, E. O.; SCHOEMAN, A. T. S.; BRITS, J. A.; MERWE, M. V. D. Laboratory evaluation of lufenuron on immature stages of potato tuber moth (Lepidoptera: Gelechiidae). Journal of Economic Entomology, Lanhan, v. 93, n. 6, p. 1741-1743, Dec. 2000. 
EPSTEIN, E. The anomaly of silicon in plant biology. Proceedings National of Academy Science, Washington, v. 91, n. 1, p. 11-17, Jan. 1994.

GOMEZ, S. A.; ÁVILA, C. J. Controle químico da lagartado-cartucho, Spodoptera frugiperda (J. E. Smith, 1797), na cultura do trigo. Dourados: EMBRAPA Agropecuária Oeste, 2001. 18 p. (Boletim de Pesquisa e Desenvolvimento).

GOUSSAIN, M. M.; MORAES, J. C.; CARVALHO, J. G.; NOGUEIRA, N. L.; ROSSI, M. L. Efeito da aplicação de silício em plantas de milho no desenvolvimento biológico da lagarta-do-cartucho Spodoptera frugiperda (J. E. Smith) (Lepidoptera: Noctuidae). Neotropical Entomology, Londrina, v. 31, n. 2, p. 305-310, abr./jun. 2002.

OLIVEIRA, L. J.; PARRA, J. R. P.; CRUZ, I. Nutrição quantitativa de lagarta-do-cartucho em milho cultivado para três níveis de alumínio. Pesquisa Agropecuária Brasileira, Brasília, v. 25, p. 235-241, fev. 1990.

SARMENTO, R. de A.; AGUIAR, R. W. de S.; AGUIAR, R. de A. S. de S.; VIEIRA, S. M. J.; OLIVEIRA, H. G. de; HOLTZ, A. M. Revisão da biologia, ocorrência e controle de Spodoptera frugiperda (Lepidoptera, Noctuidae) em milho no Brasil. Bioscience Journal, Uberlândia, v. 18, n. 2, p. 41-48, dez. 2002.

SCHMIDT, F. B. Linha básica de suscetibilidade de Spodoptera frugiperda (Lepidoptera: Noctuidade) a Lufenuron na cultura do milho. 2002. 48 f. Dissertação (Mestrado) - Escola Superior de Agricultura Luiz de Queiroz, Piracicaba, 2002.

SILVA, D. F. Biocombustíveis e produção animal impulsionarão a cultura. Agrianual, São Paulo, p. 373-374, 2004.

SILVA, P. H. S. da. Avaliação de danos de Spodoptera frugiperda (J. E. Smith) (Lepidoptera: Noctuidade) no milho cultivado com dois níveis de fertilidade. 1995. $84 \mathrm{f}$. Tese (Doutorado) - Escola Superior de Agricultura Luiz de Queiroz, Piracicaba, 1995.

SILVEIRA, L. C. P.; VENDRAMIM, J. D.; ROSSETTO, C. J. Não-preferência para alimentação da lagarta-do-cartucho em milho. Bragantia, Campinas, v. 57, 1998.

VENDRAMIM, J. D. A resistência de plantas e o manejo de pragas. In: CRÓCOMO, W. B. (Ed.). Manejo integrado de pragas. São Paulo: UNESP, 1990. p. 177-197. 